\title{
Synchronus paraspinal and hepatic hydatid disease ; a rare presentation
}

\author{
Dr.Ashish S Motewar ${ }^{1}$ Dr.Sayalee S Narwade ${ }^{2}$ Dr.Nikhil S Bhamare ${ }^{3}$ \\ Dr.Mandar R Tilak ${ }^{4}$ \\ 1) Associate professor 2) Resident 3) Resident 4)Assistant professor, \\ Dept. Of Surgery Goernment Medical College Nanded, Maharashtra,India .
}

\begin{abstract}
Hydatid cystic disease is a common zoonotic infection in Indian subcontinent.However in following case we report a rare presentation found in a young patient synchronously in paraspinal \& intrahepatic location without intercommunication.Hydatid disease is to Echinococcus species commonly granulosus, sometimes multilocularis. The common locations of hydatid cyst are the liver (65\% to $75 \%$ )and lungs (25\%-30\%).Hydatid disease rarely developes in some locations such as spleen,kidney, bones, heart,brain,peritoneum, myocardium and muscles(1-4\%).A 25 year old male presented with clo backache on right \& fullness in right paraspinal region. On clinical examination he had non ballotable lump in right paraspinal region within intra muscular plane.CT abdomen $(P+C)$ revealed well defined hypodence nonenhancing cystic lesion seen in right lobe of liver with peripheral calcification with lesion of similar morphology in right paraspinal muscles. Exploration of right paraspinal region done. Cyst was beneath oblique \& lattisimus dorsi,superficial to psoas muscle without invasion in it.Cyst opened multiple daughter cysts along with pus evacuated. Cyst excised in totto without spillage. Hydatid cyst is frequently asymptomatic, most prevalent in sheep and cattle-breeding areas.The cysts can not easily grow in muscles due to their contractility and lactic acid content. Surgery is the optimal treatment for hydatid cyst. Open cyst evacuation is indicated for gharbi types 4\&5,posterior cysts,central cysts,more than 3 cysts,infected cysts,biliary communication,pulmonary communication\&peritoneal rupture.Alternative therapies with non-toxic scolocidal agents or combination chemotherapy has been advocated in the management of recurrence and high risk of contamination.
\end{abstract}

Keywords: Hydatid,paraspinal, surgery

\section{Introduction}

Hydatid disease is due to Echinococcus species commonly granulosus sometimes multilocularis. The common locations of hydatid cyst are the liver $(65 \%$ to $75 \%)$ and lungs $\left.(25 \%-30 \%)^{[} 1,2,3\right]$. Hydatid disease rarely developes in some locations such as spleen, kidney, bones, heart, brain,peritoneum, myocardium and muscles(1$4 \%$ )[1].In our review of literature concomitant paraspinal \& intrahepatic hydatid is repored rarely.

\section{Case Report}

A 25 year old male presented with c/o backache on right since 1 month \& fullness in right paraspinal region .No h/o trauma, fever,burning micturition, pain in abdomen, weight loss, hematuria.

On clinical examination he had non ballotable lump in right paraspinal region of size $15 \times 5 \times 5 \mathrm{~cm}$ with ill defined margins intra muscular plane extending from posterior subcostal margin to iliac region with no overlying skin changes.no organomegaly.

Blood investigations including liver function tests \& kidney function tests were normal. Ultrasound examination revealed hepatomegaly with thick walled cystic lesion in right lobe of liver \& in muscle plane in right renal angle region. CT abdomen $(\mathrm{P}+\mathrm{C})$ revealed well defined hypodence nonenhancing cystic lesion of size $45 \times 40 \mathrm{~cm}$ seen in right lobe of liver with peripheral calcification with lesion of similar morphology of size $14 \times 5 \times 3.6 \mathrm{~cm}$ in right paraspinal muscles with no intercommunication between them \& no bone,spinal canal alteration or compression of right kidney.[Fig.1,2,3] 


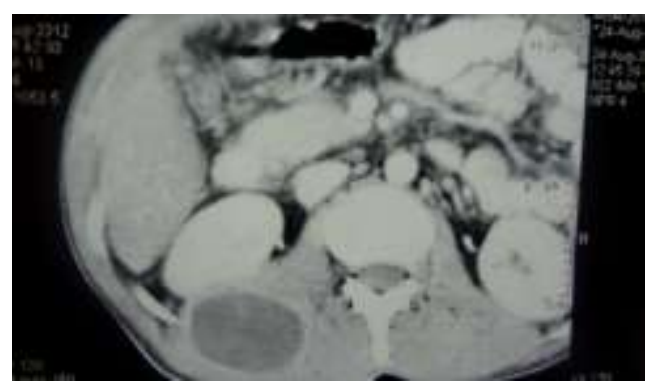

Figure: 1 shows right paraspinal hydatid

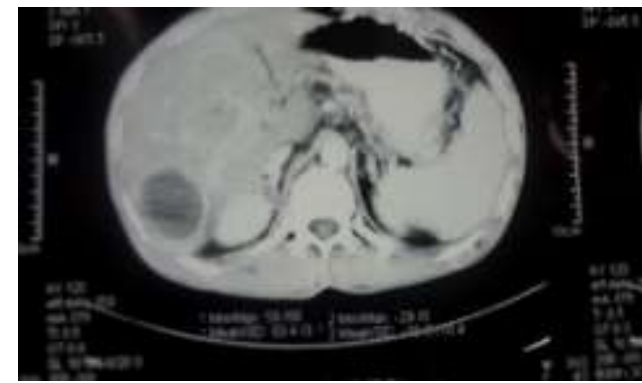

Figure :2 shows intrahepatic hydatid

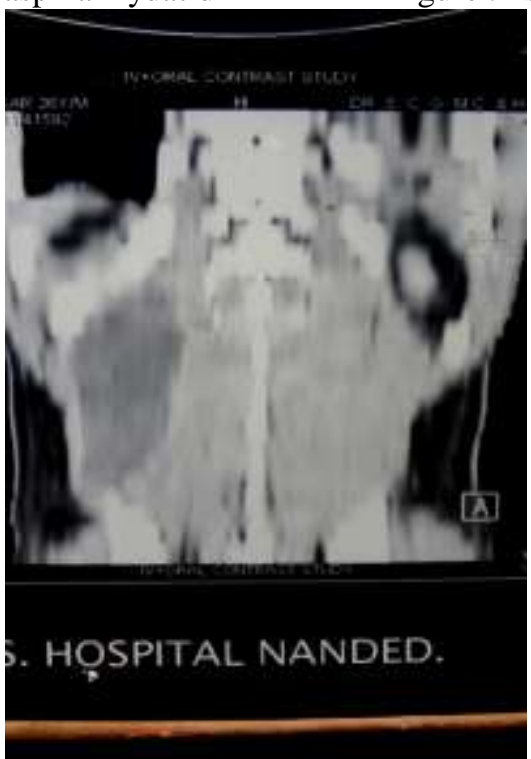

Figure:3 coronal view of CT image

So our diagnosis of paraspinal hydatid cyst was confirmed.As patient was symptomatic only for paraspinal hydatid cyst only \& size of hepatic cyst was small,exploration of right paraspinal region done after 21 day of antiscolicidal treatment. There was cyst of size $15 \times 5 \times 5 \mathrm{~cm}$ beneath oblique \& lattisimus dorsi , superficial to psoas muscle without invasion in it[Fig.4] .Cyst opened multiple daughter cysts along with pus evacuated.Cyst excised in totto without rupture \& spillage[Fig.5].Negative suction drain was kept.Post operatively on day 3 drain was removed,stitches removed day10 \&discharged.Histopathology confirmed diagnosis.

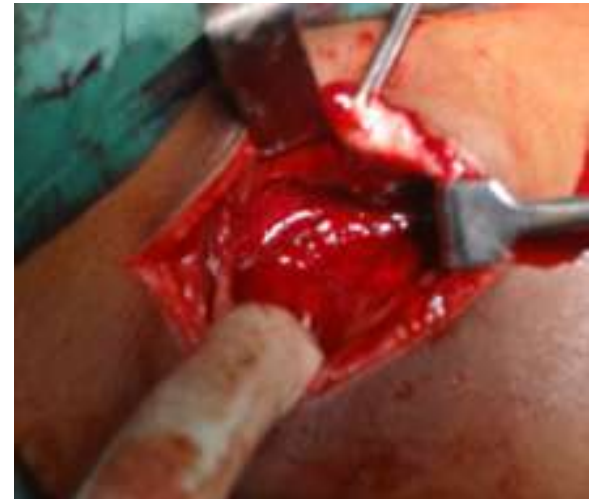

Fig.4; Intraoperrative photograph

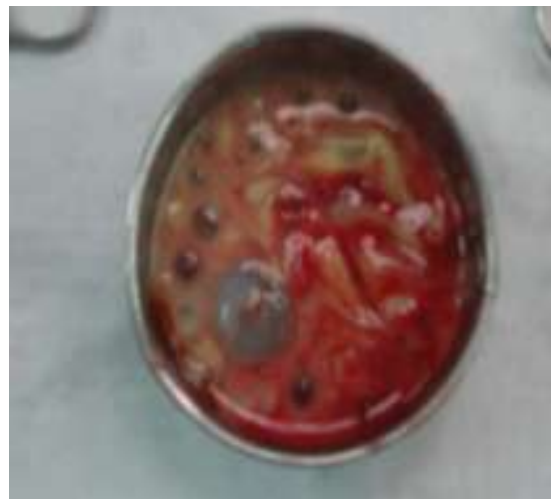

Fig.5 Photogr. Of multiple daughter cysts

III. Discussion

Hydatid disease is most prevalent in sheep- and cattle-breeding areas, where the first step in chain of transmission occurs.

The causative agent is introduced to the $\operatorname{dog}$ (the primary host) through the faeces of livestock. The minute larval form of E. granulosus lives in the small intestine of the dog species. The eggs are passed in the faeces of an infected dog and can be transferred to mammal(man -intermediate host) that ingests them.After ingestion,the 
embryos are released from the eggs, that traverse the intestinal mucosa, and disseminate systemically via venous and lymphatic channels \&develop into hydatid cysts in various body parts.

The cysts can not easily grow in muscles due to their contractility and lactic acid content.The wall of cyst in the muscle is formed by three layers, the inner germina,intermediate, and outer granulomatous adventitial layer. The most common skeletal sites include hip \& thigh,shoulder \&humerus regions.

Hydatid cyst is frequently asymptomatic[1].The latent period of cyst development varies between 5 and 20 years $[4,5]$.

Serological tests are widely used to diagnose hydatid cyst.However, positive serological results do not confirm nor negative results exclude the disease $[1,6]$.

The imaging features of hydatid cysts are well described in the literature.US is a sensitive,safe, noninvasive method, it is the procedure of choice for the diagnosis of cysts with a "honeycomb" pattern(type 3), as observed in our patient.Gharbi's classification provides morphological description on ultrasound.

Type 1-pure fluid collection,type 2-fluid collection with split wall (floating membrane),type 3-fluid collection with septa,type 4-heterogenous echographic pattern,type 5-reflecting thick walls.

As seen in our case the cyst fluid appears anechoic at US, yields an attenuation value of 3-30HU at CT,Calcifications in the cyst wall as in our case are best detected on CTscans. CT has the advantage of detecting smaller cysts when located outside the liver,and sometimes differentiating parasitic from nonparasitic cysts \& for follow-up studies during chemotherapy.Other diagnostic means such as fine needle aspiration should be avoided because of dangerous anaphylactic reactions[7].

Surgery is the optimal treatment for hydatid cyst.

Open cyst evacuation is indicated for gharbi types $4 \& 5$,posterior cysts,central cysts,more than 3 cysts,large cysts,heavy calcification,infected cysts with above criteria,biliary communication,pulmonary communication\&peritoneal rupture.

Laparoscopic evacuation is indicated in Gharbi type 1 or 2,anterior cysts,peripheral cysts,1-3 cysts,small cysts,no or minimal calcification.Pericystectomy is complete resection of cyst wall without entering the cyst cavity.

Alternative therapies with non-toxic scolocidal agents or combination chemotherapy using imidazole derivatives,particularly albendazol, has been advocated in the management of patients with recurrence and high risk of contamination[8].

\section{Conclusion}

As hydatid cyst at paraspinal location mimics soft tissue tumor,psoas abcess;so high index of suspicion is necessary for diagnosis.

\section{References}

[1] Garcia-Diez A.I., Ros Mendoza L.H.,Villacampa V.M.et al.: MRI evaluation of softtissue hydatid disease. Eur Radiol,2000, 10: 462466.

[2] White C Jr, Weller PF. Echinococcosis. In: Braunwald E, FauciAS, Kasper DL, Longo DL, Jameson JL eds Harrison's Principlesof Internal Medicine 15th edition.McGraw Hill; 2001; p.1250.

[3] Tatari H, Baran O, Anlidag T, et al. Primary intramuscular hydatidosis of supraspinatus muscle. Arch Orthop Trauma Surg.2001;121: 93-948.

[4] Berrada S., Ridai M., Mokhtari M.:Kystes Hydatiques de la rate: splénectomies ou chirurgie conservatrice Ann Chir, $1991,45: 434$.

[5] Bellakhdar A., Lamhamdi A.,Touzani K.et al.: Les kystes hydatiques de la rate (25 cas). J Chir,1986,.123: 326.

[6] Akhan O., Ensari S., Özmen M.:Percutaneos treatment of a parotid gland hydatid cyst: a possible alternativeto surgery.Eur Radiol, 2000,12: 597-599.

[7] essaki.o; hajjam M.,Kadiri R;hydatique des parties molles;aspects radiologiques:annals of radio 1996

[8] Akal M., Kara M.: Primary hydatid cyst of the posterior cervical triangle.J Laryngol Otol, 202, 116: $153-155$. 